\title{
Correlaciones entre las propiedades mecánicas del concreto reforzado con fibras de acero
}

\section{Correlations Between Mechanical Properties of Steel Fiber Reinforced Concrete}

\author{
Carrillo Julián \\ Departamento de Ingeniería Civil \\ Universidad Militar Nueva Granada, Bogotá, Colombia \\ Correo:wjcarrillo@gmail.com \\ Aperador William \\ Facultad de Ingeniería \\ Universidad Militar Nueva Granada, Bogotá, Colombia \\ Correo:wiliam.aperador@unimilitar.edu.co
}

\author{
González Giovanni \\ Departamento de Ingeniería Civil \\ Universidad Militar Nueva Granada, Bogotá, Colombia \\ Correo:gonzalez.giovani@gmail.com
}

Información del artículo: recibido: julio de 2012, aceptado: septiembre de 2012

\section{Resumen}

La resistencia a tensión y la capacidad de deformación post-agrietamiento que exhibe el concreto reforzado con fibras de acero (CRFA), impulsan su uso en elementos controlados por deformaciones de cortante. Con el propósito de desarrollar ayudas de diseño que promuevan la utilización de CRFA como refuerzo a cortante en el alma de muros de concreto para vivienda de interés social (VIS), se llevó a cabo un estudio experimental para caracterizar las propiedades mecánicas del CRFA. El programa experimental incluyó el ensayo de 128 especímenes en forma de cilindros y vigas. De acuerdo con los lineamientos especificados por ACI-318, con el espesor de los muros utilizados en VIS, y con los resultados de investigaciones previas, se seleccionaron tres fibras Dramix con relaciones longitud-diámetro de 55, 64 y 80. La dosificación de las fibras se expresó como porcentaje de la dosificación mínima que se especifica en ACI-318, cuando se desea sustituir el refuerzo mínimo convencional por cortante en vigas $\left(60 \mathrm{~kg} / \mathrm{m}^{3}\right)$. De esta manera, se utilizaron cinco dosificaciones: $0,40,45,60$ y $75 \mathrm{~kg} / \mathrm{m}^{3}$. En el estudio se determinaron las propiedades mecánicas del CRFA sometido a esfuerzos de compresión, tensión y flexión. A partir de las tendencias de los resultados experimentales, se proponen correlaciones numéricas para estimar las propiedades mecánicas básicas, y las propiedades que caracterizan el desempeño a flexión del CRFA.

\section{Descriptores:}

- concreto reforzado con fibras

- fibras de acero

- vivienda

- propiedades mecánicas

- compresión

- tensión

- flexión

- tenacidad 


\begin{abstract}
Tension strength and post-cracking deformation capacities that exhibits steel fiber reinforced concrete (SFRC) stimulate its use in elements governed by shear deformations. Aimed at developing design aids that promote the use of SFRC as web shear reinforcement of concrete walls for low-rise economic housing (LEH), an experimental study for describing the mechanical properties of SFRC was carried out. The experimental program included testing of 128 cylinder- and beam-type specimens. According to requirements specified by ACI-318, to thickness of walls used in LEH, and to results of previous studies, three Dramix fibers with length-diameter ratios of 55, 64 and 80 were selected. Fiber dosage was expressed in terms of the minimum fiber dosage specified by ACI-318 for replacing the minimum area of conventional shear reinforcement in beams $\left(60 \mathrm{~kg} / \mathrm{m}^{3}\right)$. Therefore, five dosages were used: $0,40,45,60$ and $75 \mathrm{~kg} / \mathrm{m}^{3}$. Mechanical properties of SFRC under compressive, tensile and flexural stresses were evaluated in this study. Based on trends of experimental results, numerical correlations for estimating both basic mechanical properties and properties that describe flexural performance of SFRC are proposed.
\end{abstract}

\section{Introducción}

Una de las opciones más eficientes para la construcción de viviendas de interés social (VIS), es el desarrollo de conjuntos habitacionales con viviendas de concreto en su totalidad. Teniendo en cuenta la resistencia inherente de las estructuras con muros de concreto, las demandas sísmicas en este tipo de viviendas son bajas. Por tanto, actualmente se utilizan muros con baja resistencia de concreto, así como espesor y cuantías de refuerzo reducidas. Con el propósito de incrementar y mejorar la oferta tecnológica de las viviendas a base de muros de concreto, manteniendo una seguridad estructural adecuada, Carrillo y Alcocer (2012) llevaron a cabo un programa de investigación experimental y analítico. A partir del análisis de la información experimental y analítica, se comprobó que las cuantías mínimas de refuerzo a cortante estipuladas en el Reglamento ACI-318 (2011), son conservadoras o muy conservadoras, especialmente para estructuras situadas en algunas zonas de amenaza sísmica baja o moderada. Con base en lo anterior, en dicho estudio se han presentado recomendaciones de acuerdo con la capacidad y la demanda de las viviendas. Por ejemplo, en algunas zonas se ha propuesto prescindir o disminuir el refuerzo a cortante en el alma del muro, a cambio de utilizar requisitos específicos por cambios volumétricos y/o refuerzo por integridad estructural, así como parámetros particulares para diseño sísmico.

Actualmente se reconoce que la adición de fibras de acero en vigas de concreto reforzado incrementa la resistencia a cortante. Por ejemplo, si se adiciona una can- tidad de fibra suficiente, se puede evitar falla frágil por cortante $\mathrm{y}$, al mismo tiempo, generar comportamiento más dúctil en vigas (Adebar et al., 1997). Por tanto, el concreto reforzado con fibras de acero (CRFA) también podría convertirse en una opción eficiente para la construcción de muros de VIS. Para desarrollar ayudas de diseño que promuevan el uso del CRFA en muros de concreto de VIS, se llevó a cabo un extenso programa de investigación experimental. La campaña experimental incluyó el ensayo de 128 especímenes; 88 en forma de cilindros y 40 en forma de vigas. Las variables estudiadas fueron el tipo de fibra de acero, la dosificación de fibras y el tipo de esfuerzo aplicado. De acuerdo con los lineamientos del ACI-318 (2011), con el espesor de los muros utilizados en VIS y con los resultados de investigaciones previas (Ávila et al., 2011), se utilizaron las fibras Dramix ZP-306, RC-65/35-BN y RC-80/60-BN, con relaciones longitud-diámetro $\left(l_{f} / d_{f}\right)$ de 55,64 y 80 , respectivamente. La dosificación de las fibras se expresó como porcentaje de la dosificación mínima que se especifica en el ACI-318, cuando se desea sustituir el refuerzo mínimo convencional por cortante en vigas; es decir, $D_{f-\min }=60 \mathrm{~kg} / \mathrm{m}^{3}$. De esta manera, se utilizaron cuatro dosificaciones: $0,67,75,100$ y $125 \%$ de $D_{f-m i n}$ i es decir, 0, 40, 45, 60 y $75 \mathrm{~kg} / \mathrm{m}^{3}$. En el estudio se determinaron las propiedades mecánicas del CRFA sometido a esfuerzos de compresión, tensión y flexión. A partir de las tendencias de los resultados experimentales, se proponen correlaciones numéricas para estimar las propiedades mecánicas básicas del CRFA (resistencia máxima y deformación en compresión, módulo de elasticidad, 
relación de Poisson y resistencia a tensión indirecta), y las propiedades que caracterizan el desempeño a flexión (resistencia de agrietamiento, capacidad de deformación y capacidad de disipación de energía o tenacidad).

\section{Características del CRFA}

El uso de fibras en el concreto para proveer comportamiento adecuado antes y después de agrietamiento, ha ganado gran popularidad en las últimas décadas. Desde 1967 varios tipos de fibras se han utilizado en el concreto de forma satisfactoria, pues se han mejorado las propiedades físicas y de durabilidad del concreto. Adicionalmente, los resultados de investigaciones experimentales han demostrado la capacidad de las fibras para mejorar las propiedades mecánicas del concreto (ACI-544, 2010). Las ventajas más significativas de la adición de fibras de acero al concreto son las siguientes:

a) proveen tenacidad a flexión (capacidad de absorber energía después del agrietamiento)

b) aumentan la resistencia a tensión directa, a cortante y a torsión

c) incrementan las propiedades de resistencia a impacto y a fatiga

d) mejoran el comportamiento de contracción y flujo plástico

e) incrementan la durabilidad en ciertas condiciones climáticas (ACI-544, 1996).

A pesar de las propiedades y ventajas del CRFA, en la actualidad, la utilización de CRFA en aplicaciones estructurales tiene un papel secundario, es decir, se usa esencialmente como suplemento para controlar el agrietamiento, aumentar la resistencia al impacto y resistir la desintegración del material. En elementos estructurales solicitados por tensión axial o tensión por flexión, tales como vigas, columnas, losas de entrepiso, entre otros, el acero de refuerzo debe resistir los esfuerzos de tensión. En estos casos, el uso de fibras de acero en combinación con refuerzo convencional ha mostrado buenos resultados, por ejemplo, los estudios de Jindal (1984), Batson et al. (1984) y Craig (1987) han indicado que el CRFA incrementa la resistencia a flexión, a cortante y a torsión.

\section{Resistencia a fuerza cortante en elementos de CRFA}

El incremento que proporciona el CRFA a la resistencia a cortante y a la ductilidad se deriva de su resistencia a tensión posagrietamiento. Esta resistencia residual también tiende a reducir el tamaño y el espaciamiento de las grietas (Kwak et al., 2002). Durante el estado límite de falla por cortante de una viga de concreto reforzado, la fuerza cortante es transferida esencialmente por la trabazón de los agregados, la cual depende del espesor de la grieta. A partir del adecuado comportamiento posagrietamiento que las fibras le proporcionan al concreto, la fuerza cortante resistente se incrementa y el espesor de la grieta disminuye. Cuando se forma la grieta de cortante, se generan tensiones diagonales perpendiculares a la misma, cuya proyección vertical equilibra la fuerza cortante que actúa sobre la viga. Estas tensiones equivalen al esfuerzo necesario para "desprender" las fibras de la matriz de concreto (Turmo et al., 2008). El incremento de resistencia al cortante atribuido a las fibras no sólo depende de la cantidad de fibras y la dosificación, sino también de la relación de aspecto (Jindal, 1984) y de las condiciones de anclaje de la fibra de acero (Narayana, 1987).

A medida que se incrementa la experiencia en la utilización de CRFA, también se extienden las aplicaciones aceptadas por la comunidad de la ingeniería (ACI-544, 1996). Del mismo modo, la recopilación de información y el desarrollo de nuevas investigaciones han incrementado los datos disponibles que soportan las ventajas del CRFA en elementos estructurales. De esta manera, varios reglamentos de diseño han aceptado dichas ventajas; por ejemplo, el Reglamento ACI-318 (2011) reconoce la capacidad del CRFA para resistir fuerza cortante. Por tanto, en la sección 11.4.6.1 de ACI-318 se permite el uso del CRFA en sustitución del refuerzo mínimo por cortante en vigas, el cual se dispone de forma convencional utilizando estribos de acero. El ACI-318 permite dicha sustitución, siempre y cuando la resistencia nominal a compresión del concreto $\left(f_{c}{ }^{\prime}\right)$ sea menor que $40 \mathrm{MPa}$, el peralte de la viga sea menor que $60 \mathrm{~cm}$ y el esfuerzo cortante de diseño sea menor que $\phi 0.17 \sqrt{f_{c}^{\prime}}$ MPa. Esta recomendación del ACI-318 se basa en resultados experimentales de una extensa base de datos de vigas de concreto con y sin refuerzo convencional (Parra, 2006). Si se cumplen los límites mencionados, el refuerzo mínimo con barras convencionales se puede sustituir utilizando CRFA con dosificación de fibras mayor que $60 \mathrm{kgf} / \mathrm{m}^{3}$ y que cumpla con el criterio de aceptación que se especifica en la sección 5.6.6.2 del ACI-318, el cual se basa en el desempeño a flexión del CRFA.

\section{Desempeño a flexión del CRFA}

Debido a las dificultades para llevar a cabo pruebas de tensión directa en especímenes de concreto reforzado 
con fibras, la prueba de flexión es la más representativa en el CRFA, pues a partir de ella se puede estimar la capacidad de deformación que la fibra le proporciona al concreto después de alcanzar la resistencia máxima a flexión. En la figura 1 se presenta el comportamiento característico del concreto a flexión. La última lectura de deformación que se muestra en la figura no está asociada a la capacidad última de deformación, ya que el valor de este parámetro se establece explícitamente en la norma ASTM-C-1609 (2010) como 1/150 de la longitud del claro del espécimen $\left(l_{c}\right)$. En la sección 5.6.6.2 del Reglamento ACI-318 (2011) se especifica un criterio de aceptación basado en el desempeño a flexión, el cual debe medirse siguiendo los lineamientos de la norma ASTM-C-1609. De acuerdo con dicho criterio, se considera aceptable el CRFA para resistir fuerza cortante, siempre y cuando sus resistencias residuales asociadas a deflexiones al centro del claro equivalentes a $l_{c} / 300 \mathrm{y}$ $l_{c} / 150$, sean mayores que 90 y $75 \%$, respectivamente, de la resistencia de agrietamiento (carga de primer pico $P_{p}$, (figura 1).

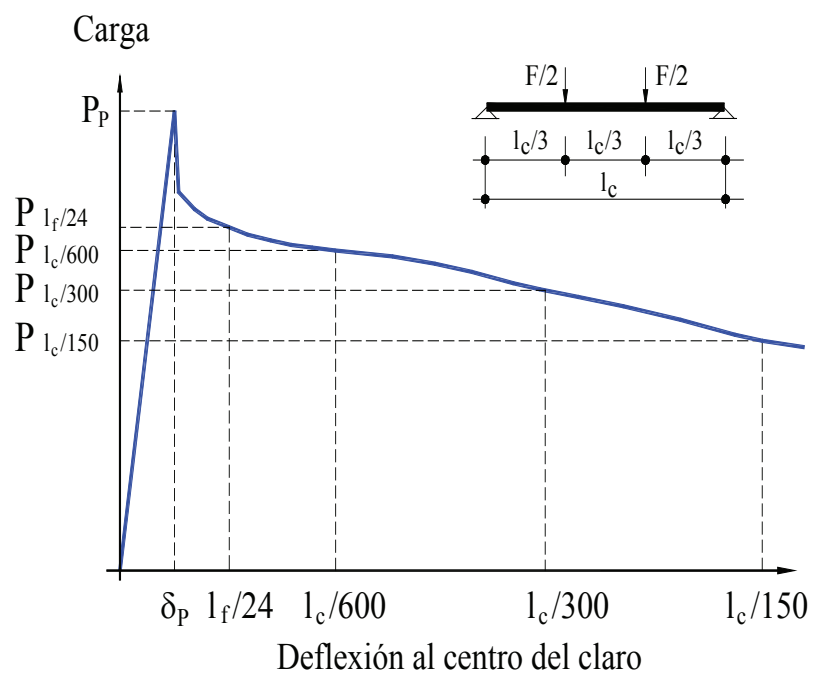

Figura 1. Curva característica carga-deflexión de CRFA

A partir de una investigación experimental donde se utilizó CRFA con diferentes longitudes de fibras, Dinh et al. (2010) han propuesto un criterio de aceptación distinto, pero basado también en la norma ASTM-C-1609; es decir, recomiendan considerar el primer límite de deflexión en función de la longitud de la fibra $1_{\mathrm{f}}$, en lugar de la longitud del claro del espécimen, $1_{c}$, así como valores diferentes de las resistencias residuales. Por ejemplo, Dinh et al. (2010) consideran aceptable un CRFA para resistir fuerza cortante, siempre y cuando sus resistencias residuales en deflexiones al centro del claro equivalentes a $1_{\mathrm{f}} / 24$ y $1_{\mathrm{c}} / 150$, no sean menores que
75 y $40 \%$, respectivamente, de la resistencia de agrietamiento (figura 1).

\section{Programa experimental}

Con el propósito de caracterizar las propiedades mecánicas básicas y las propiedades que caracterizan el desempeño a flexión del CRFA, el programa experimental incluyó el ensayo de 128 especímenes; 88 en forma de cilindros y 40 en forma de vigas.

\section{Variables de estudio}

Las variables estudiadas fueron el tipo de fibra de acero, la dosificación de fibras y el tipo de esfuerzo aplicado (tabla 1). En la sección 3.5.8 del ACI-318 (2011) se estipula que las fibras deben tener relación de aspecto (longitud-diámetro, $l_{f} / d_{f}$ ) no menor que 50 y no mayor que 100. De acuerdo con las especificaciones del ACI318, el espesor reducido de los muros para VIS y los resultados de investigaciones previas (Ávila et al., 2011), se seleccionaron tres valores de relaciones de aspecto de las fibras. Aunque ACI-318 no especifica el tipo de fibra que se debe utilizar (lisas, con gancho, onduladas o retorcidas), se seleccionó la fibra con gancho, pues se ha observado un buen desempeño a flexión (Parra, 2005) y, adicionalmente, es la más comercializada y distribuida para la construcción de elementos sometidos a efectos sísmicos. En cuanto a las dosificaciones, éstas se establecieron en función de la dosificación mínima de fibras $\left(D_{f-\text { min }}\right)$ que se especifica en la sección 11.4.6.1 del ACI-318 (2011), cuando se desea sustituir el refuerzo mínimo convencional por cortante en vigas; es decir, $D_{f-m i n}=60 \mathrm{~kg} / \mathrm{m}^{3}$. Tomando en cuenta el tipo de esfuerzos a los que se encuentran sometidos los muros de concreto para VIS, en el estudio se determinaron las propiedades mecánicas del CRFA sometido a esfuerzos de compresión, tensión y flexión.

\section{Características del concreto}

Para la construcción de los modelos se utilizaron tres concretos reforzados con fibras. Las características especificadas de los concretos se muestran en la tabla 2. Los concretos fueron premezclados y proporcionados por el Grupo CEMEX. Infortunadamente, por disposiciones del proveedor, no fue posible conocer la proporción de agregados, agua y cemento en las mezclas.

La cantidad de fibra asociada a la menor dosificación se adicionó y mezcló en planta, dentro de la revolvedora mecánica del camión transportador. El peso de fibras se calculó utilizando el valor de volumen de dise- 
Tabla 1. Descripción de las variables de estudio

\begin{tabular}{|c|c|c|c|c|c|c|c|c|}
\hline \multicolumn{2}{|r|}{ Variable } & \multicolumn{7}{|c|}{ Descripción de la variable } \\
\hline \multirow{7}{*}{ Fibra de acero } & Designación & $1 \mathrm{~F}$ & \multicolumn{3}{|c|}{$2 \mathrm{~F}$} & \multicolumn{3}{|c|}{$3 \mathrm{~F}$} \\
\hline & Referencia & ZP-305 & \multicolumn{3}{|c|}{ RC-65/35-BN } & \multicolumn{3}{|c|}{ RC-80/60-BN } \\
\hline & Tipo de anclaje & Con gancho & \multicolumn{3}{|c|}{ Con gancho } & \multicolumn{3}{|c|}{ Con gancho } \\
\hline & Longitud, $1_{\mathrm{f}}, \mathrm{mm}$ & 30 & \multicolumn{3}{|c|}{35} & \multicolumn{3}{|c|}{60} \\
\hline & Diámetro, $\mathrm{d}_{\mathrm{f}}, \mathrm{mm}$ & 0.55 & \multicolumn{3}{|c|}{0.55} & \multicolumn{3}{|c|}{0.75} \\
\hline & Relación de aspecto, $1_{\mathrm{f}} / \mathrm{d}_{\mathrm{f}}$ & 55 & \multicolumn{3}{|c|}{64} & \multicolumn{3}{|c|}{80} \\
\hline & Resistencia a tensión, $\mathrm{MPa}^{(1)}$ & 1100 & \multicolumn{3}{|c|}{1100} & \multicolumn{3}{|c|}{1100} \\
\hline \multirow{3}{*}{ Dosificación } & $\% \mathrm{D}_{\mathrm{f}-\min }$ & 67 & 75 & 100 & 125 & 75 & 100 & 125 \\
\hline & $D_{\mathrm{f}}, \mathrm{kg} / \mathrm{m}^{3}$ & 40 & 45 & 60 & 75 & 45 & 60 & 75 \\
\hline & $\mathrm{V}_{\mathrm{f}}^{(2)}, \%$ & 0.51 & 0.57 & 0.76 & 0.96 & 0.57 & 0.76 & 0.96 \\
\hline
\end{tabular}

Tipo de esfuerzo aplicado

Compresión, tensión y flexión

${ }^{(1)}$ Proporcionado por el fabricante; ${ }^{(2)} V_{f}=$ fracción de volumen $=D_{f} / \gamma_{s}$; donde $\gamma_{s}=$ densidad del acero $=7850 \mathrm{~kg} / \mathrm{m}^{3}$.

Tabla 2. Características especificadas de los tipos de CRFA

\begin{tabular}{cccc}
\hline Característica especificada & \multicolumn{2}{c}{ Tipo de fibra } \\
\cline { 2 - 4 } Tipo & $1 \mathrm{~F}$ & 2F & Peso Normal \\
\hline Resistencia a compresión, $\mathrm{f}_{\mathrm{c}}{ }^{\prime}, \mathrm{MPa}$ & Peso Normal & 20 & 30 \\
Dosificación, $\mathrm{D}_{\mathrm{f}}, \mathrm{kg} / \mathrm{m} 3$ & 20 & $45,60 \mathrm{y} 75$ & $45,60 \mathrm{y} 75$ \\
Tamaño máximo del agregado, $\mathrm{mm}$ & 40 & 10 & 10 \\
Tipo de agregado & 10 & Andesita & Andesita \\
Revenimiento, $\mathrm{cm}$ : especificado / medido & Andesita & $20 /(21,18,17)^{(1)}$ & $20 /(21,20,18)^{(1)}$ \\
\hline
\end{tabular}

(1) Medido después de la adición de fibra para cada dosificación.

ño del concreto (peso de fibras $=D_{f} \times$ volumen). Cuando finalizó la elaboración de los especímenes con determinada dosificación, se adicionó al concreto la fibra necesaria para lograr la dosificación siguiente. De forma similar a la dosificación inicial, la fibra asociada a las dosificaciones de 60 o $75 \mathrm{~kg} / \mathrm{m}^{3}$, se agregó y mezcló, en sitio, dentro de la revolvedora mecánica del camión transportador. Para garantizar la concordancia de las dosificaciones especificada y medida, el peso de fibras se calculó utilizando el valor efectivo de volumen de concreto en la revolvedora; es decir, se consideró el volumen extraído de concreto para elaborar los especímenes de las dosificaciones menores. El mezclado de las fibras se realizó siguiendo las recomendaciones del fabricante; es decir, la tasa de adición de fibras al concreto fresco fue aproximadamente igual a $40 \mathrm{~kg} / \mathrm{min}$ y el tiempo de mezclado fue el mayor entre $1 \mathrm{~min} / \mathrm{m}^{3}$ o 5 min. Tal como se esperaba, la manejabilidad del concre- to disminuyó significativamente a medida que se incrementó la cantidad de fibras en el concreto, especialmente cuando se utilizaron fibras tipo $3 \mathrm{~F}$ con longitud igual a $60 \mathrm{~mm}$.

El proceso de curado de todos los especímenes consistió en la aplicación de una membrana de curado tipo Curacreto de color blanco, con referencia JR-T1CA, la cual se aplicó siguiendo las especificaciones del fabricante.

\section{Tipos de ensayo y cantidad de especímenes}

El muestreo del concreto consistió en la obtención de cilindros de $150 \mathrm{~mm}$ de diámetro por $300 \mathrm{~mm}$ de altura, así como de vigas de $600 \mathrm{~mm}$ de longitud y $150 \mathrm{~mm}$ de alto y de ancho. Los especímenes en forma de cilindros fueron utilizados para obtener índices de resistencia a compresión, módulo de elasticidad y relación de Pois- 
son, así como resistencia a tensión por medio de ensayos de compresión simple, módulo de elasticidad y compresión diametral, respectivamente. Los especímenes en forma de vigas se utilizaron para caracterizar el desempeño a tensión por flexión del CRFA. En la tabla 3 se indican los tipos de ensayo, la norma ASTM utilizada y la cantidad de especímenes para el concreto.

\section{Configuración de ensayos}

En la figura 2 se muestra la configuración de los ensayos de módulo de elasticidad y relación de Poisson, tensión diametral y flexión. En el caso del módulo de elasticidad y la relación de Poisson, los cilindros fueron instrumentados con dos transductores de desplazamiento vertical y un deformímetro eléctrico a lo largo de su altura para determinar la deformación longitudinal promedio, así como con un transductor de desplazamiento transversal para determinar la relación de Poisson (figura 2a). En el caso de los ensayos para determinar el desempeño a tensión por flexión, se utilizó un transductor de desplazamiento para medir la deflexión en el centro del claro de los especímenes (figura 2c).

Con base en la información de la tabla 3, para el CRFA con fibra tipo $2 \mathrm{~F}$ y dosificación de $45 \mathrm{~kg} / \mathrm{m}^{3}$ se realizaron 4 ensayos de compresión simple, 3 ensayos de módulo de elasticidad, 4 ensayos de compresión diametral y 4 ensayos de flexión en vigas con cargas aplicadas en los tercios de la longitud del claro. El programa experimental de la investigación incluyó el ensayo de 128 especímenes de concreto; 88 en forma de cilindros y 40 en forma de vigas. El procedimiento, los equipos y los instrumentos de ensayo cumplieron los lineamientos especificados en las normas ASTM correspondientes.

Tabla 3. Tipos de ensayos y cantidad de especímenes

\begin{tabular}{|c|c|c|c|c|c|c|c|c|c|c|c|c|}
\hline \multirow[b]{4}{*}{ Tipo de ensayo (norma ASTM) } & \multirow{4}{*}{$\begin{array}{l}\text { Tipo de } \\
\text { espécimen }\end{array}$} & \multicolumn{9}{|c|}{ Tipo de fibra } & \multirow[b]{4}{*}{ Sub-total } & \multirow[b]{4}{*}{ Tota } \\
\hline & & \multirow{3}{*}{$\begin{array}{c}1 F \\
\begin{array}{c}D_{f^{\prime}} \\
\mathrm{kg} / \mathrm{m}^{3} \\
40\end{array}\end{array}$} & \multicolumn{4}{|c|}{$2 \mathrm{~F}$} & \multicolumn{4}{|c|}{$3 \mathrm{~F}$} & & \\
\hline & & & & & & & & & & & & \\
\hline & & & 0 & 45 & 60 & 75 & 0 & 45 & 60 & 75 & & \\
\hline Compresión (C-39) & & 6 & & 3 & 3 & 3 & 3 & 3 & 3 & 3 & 27 & \\
\hline Módulo elasticidad (C-469) & Cilindro & 6 & & 3 & 3 & 3 & 3 & 3 & 3 & 3 & 27 & 88 \\
\hline Tensión indirecta (C-496) & & 6 & & 4 & 4 & 4 & 4 & 4 & 4 & 4 & 34 & \\
\hline Tensión por flexión (C-1609) & Viga & 8 & 4 & 4 & 4 & 4 & 4 & 4 & 4 & 4 & 40 & 40 \\
\hline
\end{tabular}

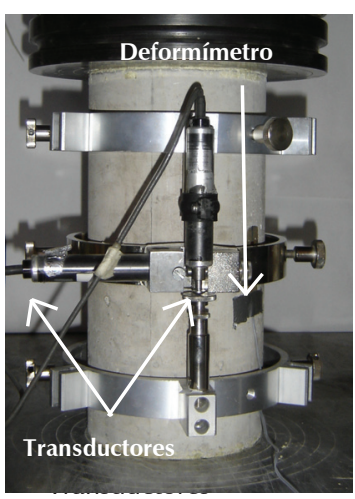

a) Elasticidad y Poisson

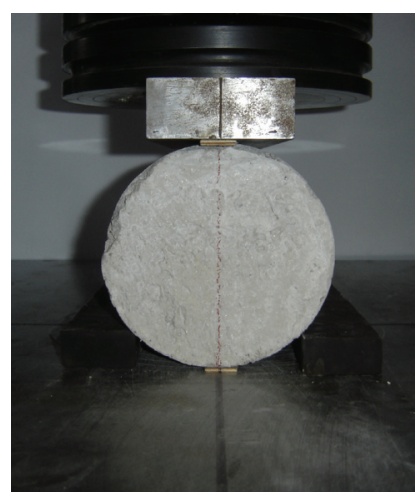

b) Tensión diametral

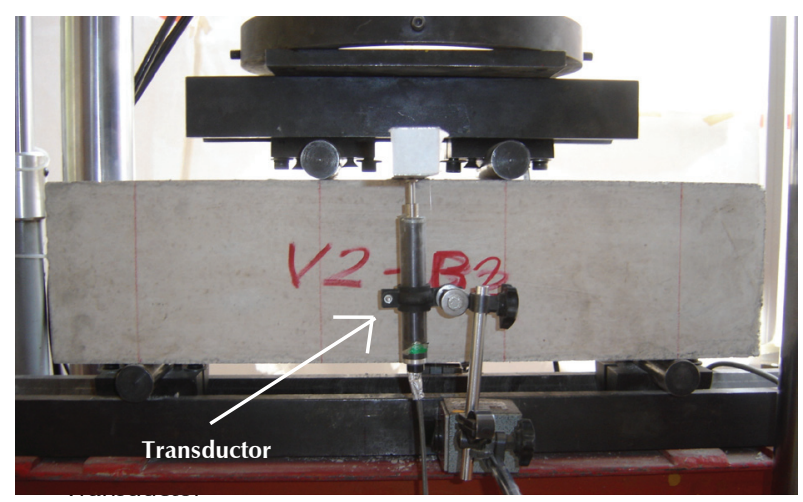

c) Flexión

Figura 2. Configuración de los ensayos 
Para aplicar la carga a los especímenes se utilizaron máquinas servo-hidráulicas con capacidad de \pm 2000 $\mathrm{kN}$ para los ensayos de compresión y tensión diametral, y de $\pm 250 \mathrm{kN}$ para los ensayos de flexión. En todos los casos, se utilizaron transductores de vástago tipo TML CDP-10, con capacidad de $\pm 5 \mathrm{~mm}$. En el caso de la deformación longitudinal de los cilindros se utilizaron deformímetros eléctricos tipo FLA-30-11-2L, con ancho de matriz de $30 \mathrm{~mm}$.

\section{Resultados experimentales y discusión}

A continuación se presentan los resultados de los ensayos de compresión simple, módulo de elasticidad, compresión diametral y flexión.
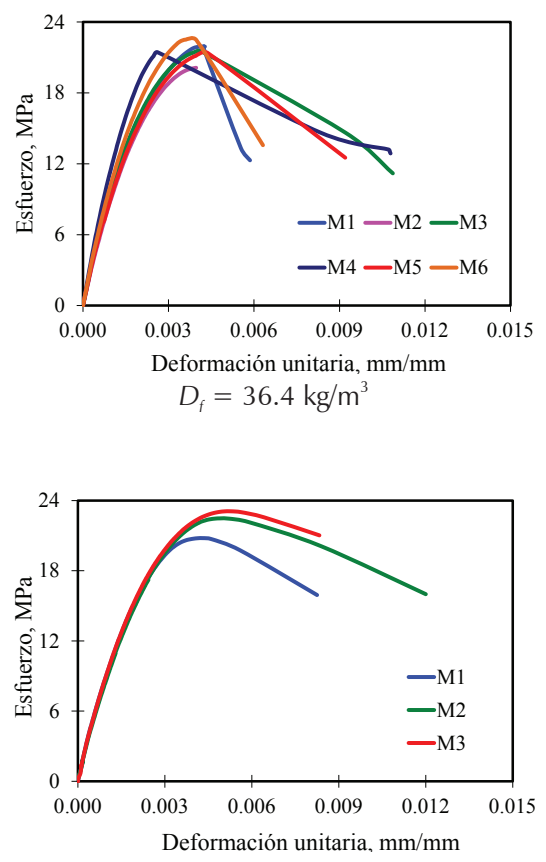

a) $\mathrm{Df}=41.2 \mathrm{~kg} / \mathrm{m3}, \mathrm{t}=93$ días

Figura 3. Curvas esfuerzo-deformación del CRFA-1F en compresión

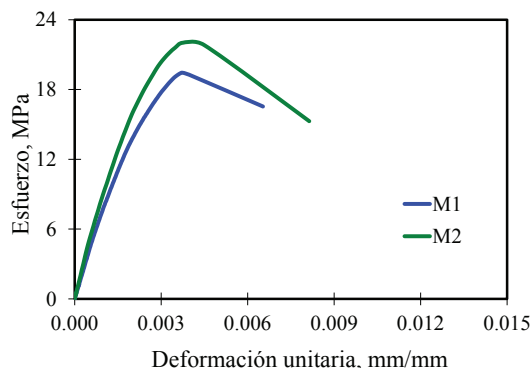

b) $\mathrm{Df}=59.3 \mathrm{~kg} / \mathrm{m} 3, \mathrm{t}=79$ días

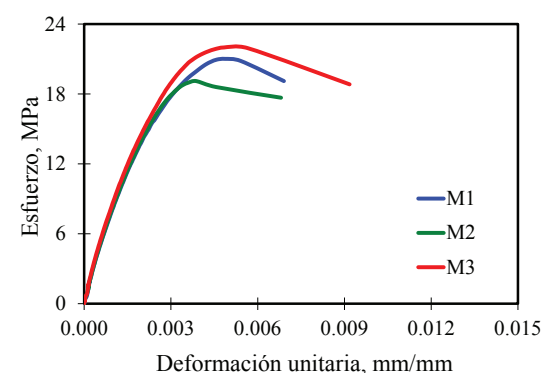

c) $\mathrm{Df}=78.6 \mathrm{~kg} / \mathrm{m3}, \mathrm{t}=50$ días

Figura 4. Curvas esfuerzo-deformación del CRFA-2F en compresión

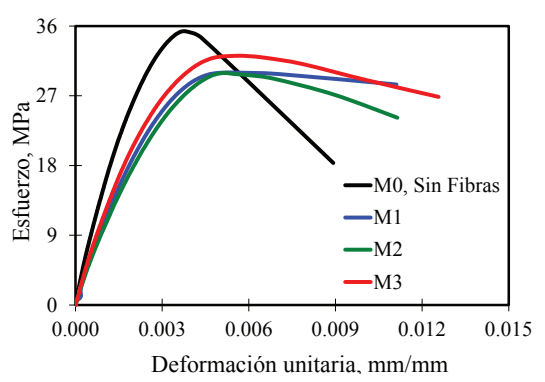

a) $\mathrm{Df}=44.2 \mathrm{~kg} / \mathrm{m} 3, \mathrm{t}=116$ días

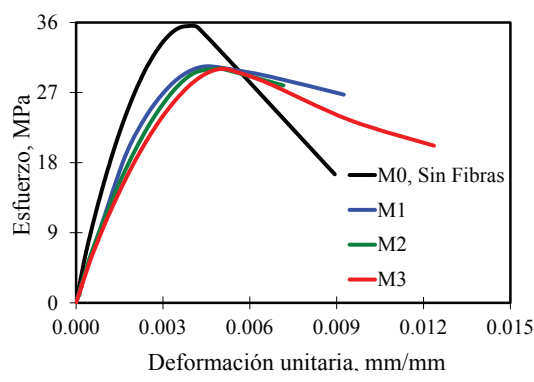

b) $\mathrm{Df}=66.1 \mathrm{~kg} / \mathrm{m} 3, \mathrm{t}=98$ días

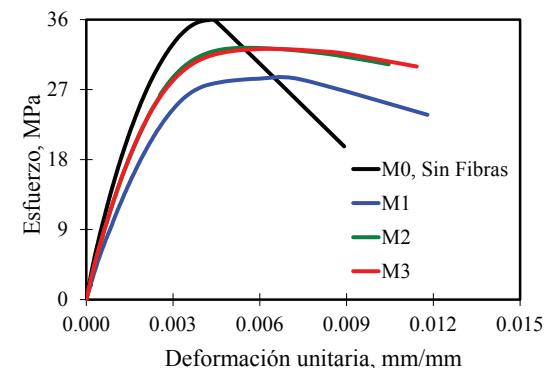

c) $\mathrm{Df}=84.0 \mathrm{~kg} / \mathrm{m} 3, \mathrm{t}=90$ días

Figura 5. Curvas esfuerzo-deformación del CRFA-3F en compresión 
En la tabla 4 se presentan las características básicas y las propiedades mecánicas promedio del concreto en compresión y en tensión. En la tabla 4 se indican la dosificación medida y la edad en la cual se determinaron las propiedades del concreto. La nomenclatura de propiedades mecánicas que se indican en la tabla 4 se describe a continuación:

$f_{c}=$ resistencia a compresión

$f_{c 0}=$ resistencia a compresión del concreto sin fibras

$\varepsilon_{0}=$ deformación unitaria en la resistencia a comisión

$E_{c}=$ módulo de elasticidad

$v=$ relación de Poisson

$f_{t}=$ resistencia a tensión (compresión diametral)

$\gamma_{c o n c}=$ peso específico del concreto en estado endurecdo.

Para extraer el efecto de la variación de resistencias entre las tres mezclas de CRFA, se obtuvo el cociente entre la resistencia a compresión del CRFA y la resistencia a compresión del concreto sin fibras $\left(f_{c} / f_{c 0}\right)$. De modo similar, se obtuvieron los siguientes cocientes para el módulo de elasticidad y la resistencia a tensión del concreto: $E_{c} / \sqrt{f_{c}}$ y $f_{t} / \sqrt{f_{c}}$. Para evaluar estadísticamente los resultados medidos, en la tabla se presenta el coeficiente de variación $(\mathrm{CV})$; es decir, el cociente porcentual entre la desviación estándar $(S)$ y el promedio de los datos $(X)$.

En la figura 6 se muestran las relaciones entre el producto $V_{f} \times\left(l_{f} / d_{f}\right)$ (relación de volumen de fibras, $\%, \times$ relación de aspecto de la fibra) y las propiedades mecánicas en compresión y en tensión. Para cada una de las relaciones se muestra una ecuación obtenida a partir de un análisis de regresión lineal. La precisión de las ecuaciones se evaluó por medio del coeficiente de correlación $(r)$, el cual mide la intensidad de la relación lineal entre los valores estimados y los datos medidos experi-

Tabla 4. Características básicas y propiedades mecánicas del concreto en compresión y en tensión

\begin{tabular}{|c|c|c|c|c|c|c|c|c|}
\hline \multirow{3}{*}{$\begin{array}{c}\text { Característica o } \\
\text { propiedad }\end{array}$} & \multicolumn{8}{|c|}{ Tipo de fibra } \\
\hline & \multirow{2}{*}{$\begin{array}{c}1 \mathrm{~F} \\
l_{f} / d_{f}=55 \\
36.4 \\
(11.1 \%)\end{array}$} & \multicolumn{3}{|c|}{$\begin{array}{c}2 \mathrm{~F} \\
l_{f} / d_{f}=64\end{array}$} & \multicolumn{4}{|c|}{$\begin{array}{c}3 \mathrm{~F} \\
l_{f} / d_{f}=80\end{array}$} \\
\hline & & $\begin{array}{c}41.2 \\
(6.6 \%)\end{array}$ & $\begin{array}{c}59.3 \\
(5.4 \%)\end{array}$ & $\begin{array}{c}78.6 \\
(5.0 \%)\end{array}$ & 0 & $\begin{array}{c}44.2 \\
(2.6 \%)\end{array}$ & $\begin{array}{c}66.1 \\
(3.2 \%)\end{array}$ & $\begin{array}{c}84.0 \\
(4.4 \%)\end{array}$ \\
\hline$V_{f}$ medida, \% & $\begin{array}{c}0.46 \\
(11.1 \%)\end{array}$ & $\begin{array}{c}0.52 \\
(6.6 \%)\end{array}$ & $\begin{array}{c}0.76 \\
(5.4 \%)\end{array}$ & $\begin{array}{c}1.00 \\
(5.0 \%)\end{array}$ & 0 & $\begin{array}{c}0.56 \\
(2.6 \%)\end{array}$ & $\begin{array}{c}0.84 \\
(3.2 \%)\end{array}$ & $\begin{array}{c}1.07 \\
(4.4 \%)\end{array}$ \\
\hline Edad, $t$, días & 80 & 93 & 79 & 50 & 101 & 116 & 98 & 90 \\
\hline$\gamma_{\text {conc }}, \mathrm{kg} / \mathrm{m}^{3}$ & $\begin{array}{c}1980 \\
(1.8 \%)\end{array}$ & $\begin{array}{c}1920 \\
(2.6 \%)\end{array}$ & $\begin{array}{c}1930 \\
(0.2 \%)\end{array}$ & $\begin{array}{c}1930 \\
(0.9 \%)\end{array}$ & $\begin{array}{c}2020 \\
(0.5 \%)\end{array}$ & $\begin{array}{c}2010 \\
(1.2 \%)\end{array}$ & $\begin{array}{c}2010 \\
(1.9 \%)\end{array}$ & $\begin{array}{c}2040 \\
(0.6 \%)\end{array}$ \\
\hline$f_{c}, \mathrm{MPa}$ & $\begin{array}{c}21.4 \\
(2.8 \%)\end{array}$ & $\begin{array}{c}22.2 \\
(3.8 \%)\end{array}$ & $\begin{array}{c}21.0 \\
(5.4 \%)\end{array}$ & $\begin{array}{c}20.3 \\
(6.3 \%)\end{array}$ & $\begin{array}{c}35.6 \\
(0.7 \%)\end{array}$ & $\begin{array}{c}31.1 \\
(3.6 \%)\end{array}$ & $\begin{array}{c}30.8 \\
(3.5 \%)\end{array}$ & $\begin{array}{c}30.7 \\
(5.4 \%)\end{array}$ \\
\hline$f_{c} / f_{c o}$ & --- & --- & --- & --- & 1.00 & 0.87 & 0.86 & 0.86 \\
\hline$\varepsilon_{0}$ & $\begin{array}{c}0.0038 \\
(15.6 \%)\end{array}$ & $\begin{array}{l}0.0048 \\
(8.2 \%)\end{array}$ & $\begin{array}{l}0.0037 \\
(0.3 \%)\end{array}$ & $\begin{array}{c}0.0045 \\
(10.2 \%)\end{array}$ & $\begin{array}{l}0.0039 \\
(5.5 \%)\end{array}$ & $\begin{array}{l}0.0054 \\
(3.5 \%)\end{array}$ & $\begin{array}{l}0.0048 \\
(4.6 \%)\end{array}$ & $\begin{array}{l}0.0060 \\
(6.2 \%)\end{array}$ \\
\hline$E_{c}, \mathrm{MPa}$ & $\begin{array}{c}10367 \\
(10.7 \%)\end{array}$ & $\begin{array}{c}9050 \\
(3.5 \%)\end{array}$ & $\begin{array}{l}8508 \\
(7.7 \%)\end{array}$ & $\begin{array}{c}8337 \\
(1.9 \%)\end{array}$ & $\begin{array}{l}15857 \\
(1.4 \%)\end{array}$ & $\begin{array}{l}10611 \\
(7.3 \%)\end{array}$ & $\begin{array}{l}10615 \\
(5.6 \%)\end{array}$ & $\begin{array}{c}12384 \\
(10.8 \%)\end{array}$ \\
\hline$E_{c} / \sqrt{f_{c}}, \mathrm{MPa}$ & $\begin{array}{c}2236 \\
(11.1 \%)\end{array}$ & $\begin{array}{c}1927 \\
(4.9 \%)\end{array}$ & $\begin{array}{c}1867 \\
(4.6 \%)\end{array}$ & $\begin{array}{c}1835 \\
(3.5 \%)\end{array}$ & $\begin{array}{c}2658 \\
(1.4 \%)\end{array}$ & $\begin{array}{c}1914 \\
(5.9 \%)\end{array}$ & $\begin{array}{c}1933 \\
(5.5 \%)\end{array}$ & $\begin{array}{c}2220 \\
(8.0 \%)\end{array}$ \\
\hline$v$ & $\begin{array}{c}0.13 \\
(11.6 \%)\end{array}$ & $\begin{array}{c}0.15 \\
(7.1 \%)\end{array}$ & $\begin{array}{c}0.14 \\
(2.3 \%)\end{array}$ & $\begin{array}{c}0.17 \\
(9.9 \%)\end{array}$ & $\begin{array}{c}0.21 \\
(5.8 \%)\end{array}$ & $\begin{array}{c}0.16 \\
(3.4 \%)\end{array}$ & $\begin{array}{c}0.14 \\
(7.1 \%)\end{array}$ & $\begin{array}{c}0.15 \\
(5.0 \%)\end{array}$ \\
\hline$f_{t}, \mathrm{MPa}$ & $\begin{array}{c}2.00 \\
(14.2 \%)\end{array}$ & $\begin{array}{c}2.47 \\
(6.7 \%)\end{array}$ & $\begin{array}{c}2.32 \\
(3.3 \%)\end{array}$ & $\begin{array}{c}2.63 \\
(7.0 \%)\end{array}$ & $\begin{array}{c}2.13 \\
(7.7 \%)\end{array}$ & $\begin{array}{c}3.60 \\
(4.4 \%)\end{array}$ & $\begin{array}{c}4.27 \\
(10.2 \%)\end{array}$ & $\begin{array}{c}4.40 \\
(7.7 \%)\end{array}$ \\
\hline$f_{t} / \sqrt{f_{c}}, \mathrm{MPa}$ & $\begin{array}{c}0.43 \\
(14.2 \%)\end{array}$ & $\begin{array}{c}0.52 \\
(6.7 \%)\end{array}$ & $\begin{array}{c}0.51 \\
(3.3 \%)\end{array}$ & $\begin{array}{c}0.58 \\
(7.0 \%)\end{array}$ & $\begin{array}{c}0.36 \\
(7.7 \%)\end{array}$ & $\begin{array}{c}0.65 \\
(4.4 \%)\end{array}$ & $\begin{array}{c}0.77 \\
(10.2 \%)\end{array}$ & $\begin{array}{c}0.79 \\
(7.7 \%)\end{array}$ \\
\hline
\end{tabular}

$(C V)=$ el valor entre paréntesis indica el coeficiente de variación en porcentaje $=[S / X] \times 100$; donde: $S=$ desviación estándar, $X=$ promedio 


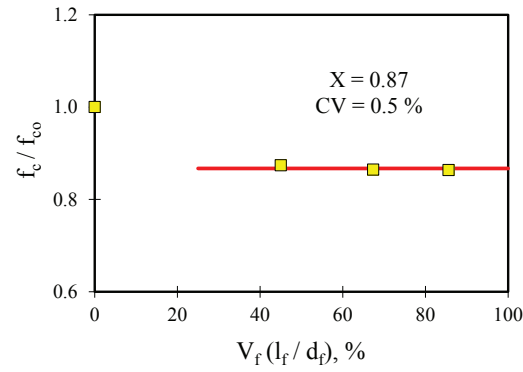

a) $f_{c} / f_{c-o}$

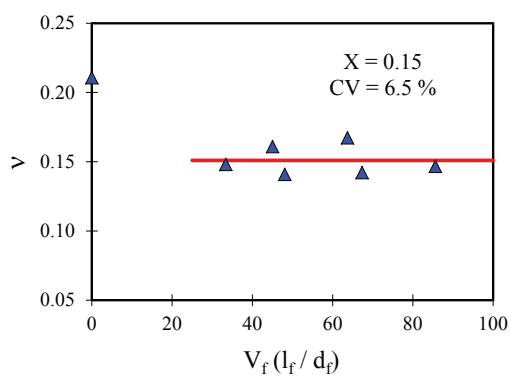

d) $v$

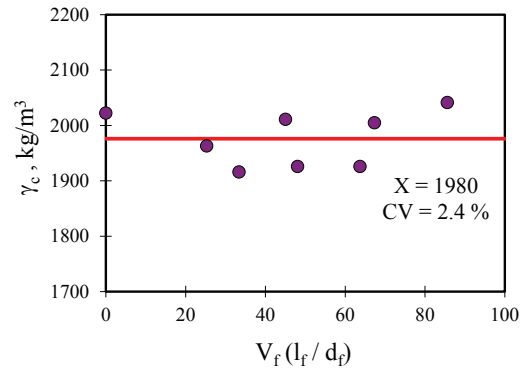

b) $\varepsilon_{0}$

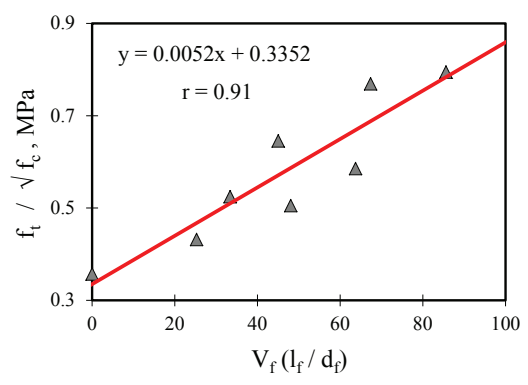

e) $f_{t}$

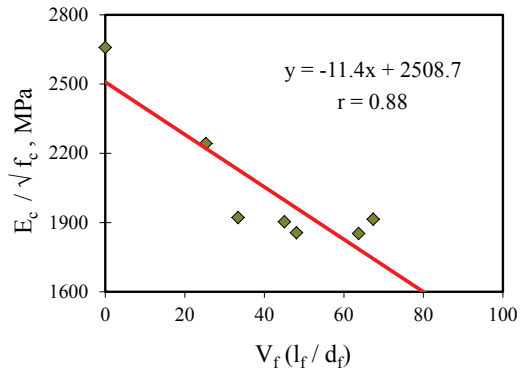

c) $E_{c}$

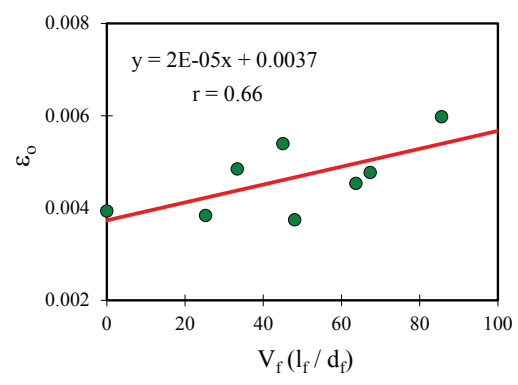

f) $\gamma_{c}$

Figura 6. Tendencias de las propiedades mecánicas en compresión y en tensión

mentalmente. En el caso de tendencias constantes, la precisión de la predicción se evaluó a partir del coeficiente de variación $(\mathrm{CV})$.

En las figuras $6 a$ y $6 \mathrm{~b}$ se observa que a medida que incrementa el contenido de fibras de acero, la resistencia a compresión del concreto disminuye a un valor casi constante $\left(f_{c} / f_{c o}=0.87\right)$ y su capacidad de deformación en compresión aumenta. Adicionalmente, en la figura $6 b$ se observa que el módulo de elasticidad disminuye a medida que se incrementa el contenido de fibras de acero. Según Kalman (2010) y Miao et al. (2003), esta tendencia se genera por la disminución de agregado grueso (sustitución de agregado grueso por fibra de acero) y por el aumento del contenido de aire en la mezcla. Este aire queda atrapado por la disminución de revenimiento del concreto, o se introduce en el momento del mezclado de las fibras. Sin embargo, la fibra le proporciona un efecto de confinamiento al concreto en compresión y, por tanto, a medida que incrementa el contenido de fibras de acero, la relación de Poisson disminuye a un valor casi constante, $v=0.15$ (figura 6d). En términos generales, la fibra de acero ocasiona que el concreto se vuelva ligeramente menos resistente, pero más dúctil cuando se somete a fuerzas axiales de compresión. En cuanto al ensayo de compresión diametral, tal como se esperaba, la resistencia a tensión indirecta del concreto aumenta proporcionalmente con la cantidad de fibras de acero (figura 6e). En cuanto al peso específico en estado endurecido, éste no varía significativamente al aumentar el contenido de fibras, pues la disminución de agregado grueso se equilibra con la adición de fibras de acero (figura 6f).

\section{Desempeño en flexión}

Como se indicó en la sección de desempeño a flexión del CRFA, debido a las dificultades para llevar a cabo pruebas de tensión directa en especímenes de concreto, la prueba de flexión se considera una de las más representativas para el CRFA, pues a partir de ella se puede estimar la capacidad de deformación que la fibra le proporciona al concreto después de alcanzar el agrietamiento inicial por flexión. Adicionalmente, a partir de los resultados medidos durante esta prueba, se recomienda determinar la tenacidad del CRFA (ACI-544, 1996). La tenacidad es la capacidad de absorción de energía que desarrolla un material y se obtiene a partir del área bajo la curva carga-deflexión medida en un espécimen de viga sometido a cargas puntuales en los tercios, tal como se establece en la norma ASTM-C-1609 (2010). En las figuras 7, 8 y 9 se presentan las curvas carga-deflexión de los concretos reforzados con fibras 
tipo $1 \mathrm{~F}, 2 \mathrm{~F}$ y $3 \mathrm{~F}$, respectivamente, junto con la curva carga-deflexión del concreto sin fibras (espécimen de control). Para el caso de la fibra $1 \mathrm{~F}$, no se obtuvieron especímenes en forma de viga de concreto sin fibras (tabla 3). En las figuras se indica la dosificación real (medida) de fibras de acero.

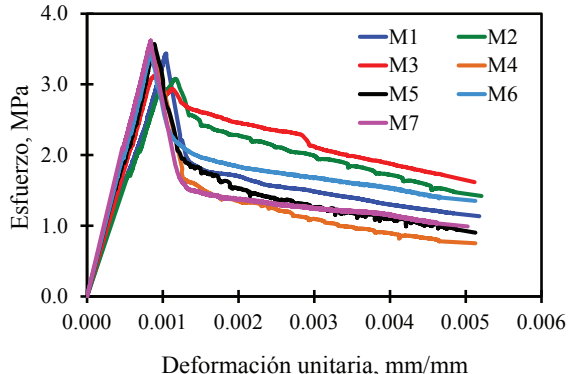

$D_{f}=36.4 \mathrm{~kg} / \mathrm{m}^{3}$

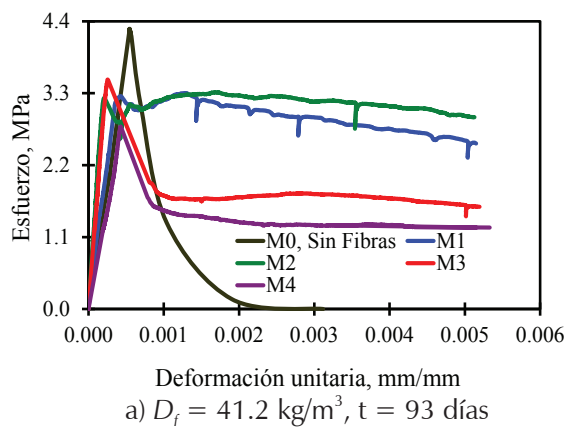

Figura 8. Curvas esfuerzo-deformación del CRFA-2F en flexión

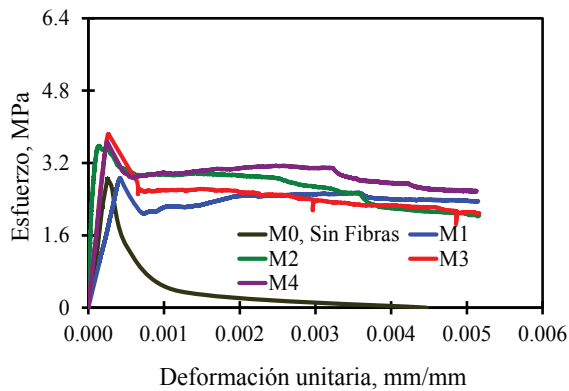

a) $D_{f}=44.2 \mathrm{~kg} / \mathrm{m}^{3}, t=116$ días
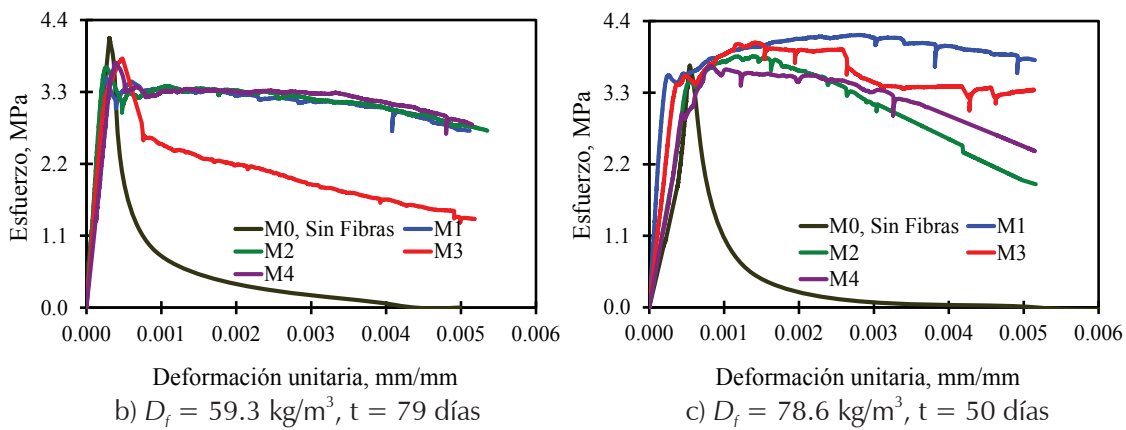

Figura 7. Curvas esfuerzo-deformación del CRFA-1F en flexión

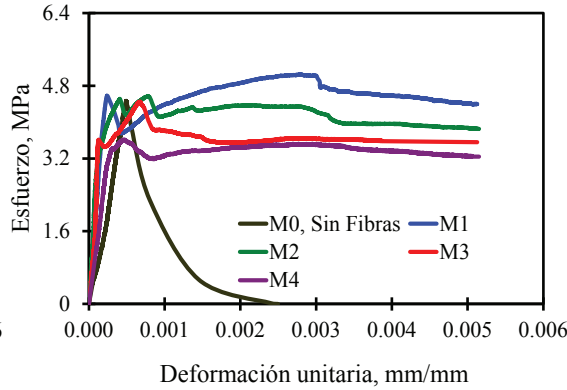

b) $D_{f}=66.1 \mathrm{~kg} / \mathrm{m}^{3}, t=98$ días

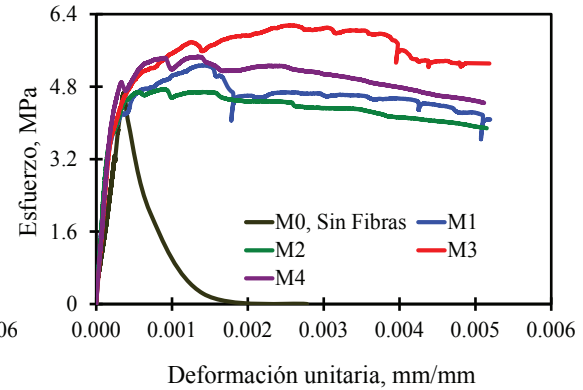

c) $D_{f}=84.0 \mathrm{~kg} / \mathrm{m}^{3}, t=90$ días

Figura 9. Curvas esfuerzo-deformación del CRFA-3F en flexión 
En la tabla 5 se presentan las propiedades mecánicas promedio del concreto sometido a esfuerzos de tensión por flexión. Las correlaciones entre estas propiedades y la raíz cuadrada de la resistencia a compresión del concreto se indican en la tablas 5. De forma similar a la tabla 4 , en la tabla 5 se presenta el coeficiente de variación de los datos medidos $(\mathrm{CV})$. La nomenclatura de propiedades mecánicas que se indican en la tabla 5 se describe a continuación:

$f_{r}=$ resistencia al agrietamiento de tensión por flexión (módulo ruptura)

$\varepsilon_{r}=$ deformación unitaria asociada a $f_{r}$

$f_{\max }=$ resistencia máxima de tensión por flexión

$\varepsilon_{\max }=$ deformación unitaria asociada a $f_{\max }$

$f_{l f / 24}=$ resistencia residual de tensión por flexión asociada a una deflexión en el centro del claro equivalente a $l_{f} / 24$ $f_{l c / 300}=$ resistencia residual de tensión por flexión aso ciada a una deflexión en el centro del claro equi valente a $l_{c} / 300$

$f_{l c / 150}=$ resistencia residual de tensión por flexión asociada una a deflexión en el centro del claro equivalente a $l_{c} / 150$

$T=$ tenacidad en Joule $(\mathrm{J})$.

Para extraer el efecto de la variación de resistencias entre las tres mezclas de CRFA, se obtuvieron los cocientes entre la resistencia al agrietamiento o la resistencia máxima y la raíz cuadrada de la resistencia a compresión del CRFA $\left(f_{r} / \sqrt{f_{c}}, f_{\max } / \sqrt{f_{c}}\right)$. De modo similar, se obtuvieron los cocientes entre las resistencias residuales y el módulo de ruptura del CRFA $\left(f_{l f / 24} / f_{r^{\prime}} f_{l c / 300} / f_{r}, f_{l c / 150} / f_{r}\right)$.

Tabla 5. Propiedades mecánicas del concreto en tensión indirecta y en tensión por flexión

\begin{tabular}{|c|c|c|c|c|c|c|c|c|c|}
\hline \multirow{3}{*}{$\begin{array}{l}\text { Característica o } \\
\text { propiedad } \\
\mathrm{D}_{\mathrm{f}} \text { medida, } \mathrm{kg} / \mathrm{m}^{3}\end{array}$} & \multicolumn{9}{|c|}{ Tipo de fibra } \\
\hline & \multirow{2}{*}{\begin{tabular}{l}
\multicolumn{1}{c}{$1 \mathrm{~F}$} \\
$\mathrm{I}_{\mathrm{f}} / \mathrm{d}_{\mathrm{f}}=55$ \\
36.4 \\
$(11.1 \%)$
\end{tabular}} & \multicolumn{4}{|c|}{$\begin{array}{c}2 \mathrm{~F} \\
\mathrm{l}_{\mathrm{f}} / \mathrm{d}_{\mathrm{f}}=64\end{array}$} & \multicolumn{4}{|c|}{$\begin{array}{c}3 F \\
\mathrm{l}_{\mathrm{f}} / \mathrm{d}_{\mathrm{f}}=80\end{array}$} \\
\hline & & 0 & $\begin{array}{l}41.2 \\
(6.6 \%)\end{array}$ & $\begin{array}{l}59.3 \\
(5.4 \%)\end{array}$ & $\begin{array}{l}78.6 \\
(5.0 \%)\end{array}$ & 0 & $\begin{array}{l}44.2 \\
(2.6 \%)\end{array}$ & $\begin{array}{l}66.1 \\
(3.2 \%)\end{array}$ & $\begin{array}{l}84.0 \\
(4.4 \%)\end{array}$ \\
\hline$V_{f}$ medida, $\%$ & $\begin{array}{l}0.46 \\
(11.1 \%)\end{array}$ & 0 & $\begin{array}{l}0.52 \\
(6.6 \%)\end{array}$ & $\begin{array}{l}0.76 \\
(5.4 \%)\end{array}$ & $\begin{array}{l}1.00 \\
(5.0 \%)\end{array}$ & 0 & $\begin{array}{l}0.56 \\
(2.6 \%)\end{array}$ & $\begin{array}{l}0.84 \\
(3.2 \%)\end{array}$ & $\begin{array}{l}1.07 \\
(4.4 \%)\end{array}$ \\
\hline $\mathrm{f}_{\mathrm{r}}, \mathrm{MPa}$ & 3.36 & $\begin{array}{l}4.04 \\
(5.9 \%)\end{array}$ & $\begin{array}{l}3.19 \\
(8.1 \%)\end{array}$ & $\begin{array}{l}3.69 \\
(3.0 \%)\end{array}$ & $\begin{array}{l}3.35 \\
(6.9 \%)\end{array}$ & $\begin{array}{l}3.99 \\
(20.3 \%)\end{array}$ & $\begin{array}{l}3.49 \\
(10.7 \%)\end{array}$ & $\begin{array}{l}4.03 \\
(13.0 \%)\end{array}$ & $\begin{array}{l}4.37 \\
(9.5 \%)\end{array}$ \\
\hline$\varepsilon_{\mathrm{r}}$ & --- & $\begin{array}{l}0.00046 \\
(24.1 \%)\end{array}$ & $\begin{array}{l}0.00033 \\
(31.1 \%)\end{array}$ & $\begin{array}{l}0.00035 \\
(27.2 \%)\end{array}$ & $\begin{array}{l}0.00039 \\
(26.3 \%)\end{array}$ & 0.00037 & 0.00027 & 0.00027 & 0.00032 \\
\hline $\mathrm{f}_{\mathrm{r}} / \sqrt{f_{c}}, \mathrm{MPa}$ & 0.73 & --- & $\begin{array}{l}0.68 \\
(8.1 \%)\end{array}$ & $\begin{array}{l}0.80 \\
(3.0 \%)\end{array}$ & $\begin{array}{l}0.74 \\
(6.9 \%)\end{array}$ & $\begin{array}{l}0.67 \\
(20.3 \%)\end{array}$ & $\begin{array}{l}0.63 \\
(10.7 \%)\end{array}$ & $\begin{array}{l}0.73 \\
(13.0 \%)\end{array}$ & $\begin{array}{l}0.79 \\
(9.5 \%)\end{array}$ \\
\hline $\mathrm{f}_{\max }, \mathrm{MPa}$ & 3.40 & $\begin{array}{l}4.04 \\
(5.9 \%)\end{array}$ & $\begin{array}{l}3.23 \\
(8.2 \%)\end{array}$ & $\begin{array}{l}3.69 \\
(3.0 \%)\end{array}$ & $\begin{array}{l}3.96 \\
(4.6 \%)\end{array}$ & $\begin{array}{l}3.99 \\
(20.3 \%)\end{array}$ & $\begin{array}{l}3.49 \\
(10.7 \%)\end{array}$ & $\begin{array}{l}4.42 \\
(11.7 \%)\end{array}$ & $\begin{array}{l}5.41 \\
(9.4 \%)\end{array}$ \\
\hline$\varepsilon_{\max }$ & --- & $\begin{array}{l}0.00046 \\
(24.1 \%)\end{array}$ & $\begin{array}{l}0.00091 \\
(34.7 \%)\end{array}$ & $\begin{array}{l}0.00035 \\
(27.2 \%)\end{array}$ & $\begin{array}{l}0.00161 \\
(35.4 \%)\end{array}$ & $\begin{array}{l}0.00037 \\
(26.1 \%)\end{array}$ & $\begin{array}{l}0.00027 \\
(37.7 \%)\end{array}$ & $\begin{array}{l}0.00118 \\
(39.4 \%)\end{array}$ & $\begin{array}{l}0.00155 \\
(39.8 \%)\end{array}$ \\
\hline $\mathrm{f}_{\max } / \sqrt{f_{c}}, \mathrm{MPa}$ & 0.73 & --- & $\begin{array}{l}0.69 \\
(8.2 \%)\end{array}$ & $\begin{array}{l}0.80 \\
(3.0 \%)\end{array}$ & $\begin{array}{l}0.88 \\
(4.6 \%)\end{array}$ & $\begin{array}{l}0.67 \\
(20.3 \%)\end{array}$ & $\begin{array}{l}0.63 \\
(10.7 \%)\end{array}$ & $\begin{array}{l}0.80 \\
(11.7 \%)\end{array}$ & $\begin{array}{l}0.98 \\
(9.4 \%)\end{array}$ \\
\hline $\mathrm{f}_{\text {If } / 24} / \mathrm{f}_{\mathrm{r}}$ & 0.53 & 0 & $\begin{array}{l}0.72 \\
(34.1 \%)\end{array}$ & $\begin{array}{l}0.81 \\
(19.2 \%)\end{array}$ & $\begin{array}{l}1.12 \\
(7.1 \%)\end{array}$ & 0 & $\begin{array}{l}0.69 \\
(15.0 \%)\end{array}$ & $\begin{array}{l}0.96 \\
(5.3 \%)\end{array}$ & $\begin{array}{l}1.07 \\
(7.0 \%)\end{array}$ \\
\hline$f_{\mathrm{lc} / 300} / \mathrm{f}_{\mathrm{r}}$ & 0.50 & 0 & $\begin{array}{l}0.72 \\
(33.7 \%)\end{array}$ & $\begin{array}{l}0.80 \\
(19.4 \%)\end{array}$ & $\begin{array}{l}1.12 \\
(7.7 \%)\end{array}$ & 0 & $\begin{array}{l}0.79 \\
(11.1 \%)\end{array}$ & $\begin{array}{l}1.02 \\
(4.8 \%)\end{array}$ & $\begin{array}{l}1.17 \\
(8.9 \%)\end{array}$ \\
\hline $\mathrm{f}_{\mathrm{lc} / 150} / \mathrm{f}_{\mathrm{r}}$ & 0.35 & 0 & $\begin{array}{l}0.65 \\
(31.8 \%)\end{array}$ & $\begin{array}{l}0.65 \\
(26.4 \%)\end{array}$ & $\begin{array}{l}0.85 \\
(23.6 \%)\end{array}$ & 0 & $\begin{array}{l}0.66 \\
(16.9 \%)\end{array}$ & $\begin{array}{l}0.94 \\
(5.3 \%)\end{array}$ & $\begin{array}{l}1.02 \\
(9.0 \%)\end{array}$ \\
\hline$T, J$ & 37.5 & $\begin{array}{l}15.2 \\
(5.7 \%)\end{array}$ & $\begin{array}{l}52.2 \\
(28.7 \%)\end{array}$ & $\begin{array}{l}64.0 \\
(15.2 \%)\end{array}$ & $\begin{array}{l}76.1 \\
(11.4 \%)\end{array}$ & $\begin{array}{l}12.0 \\
(17.4 \%)\end{array}$ & $\begin{array}{l}59.2 \\
(8.1 \%)\end{array}$ & $\begin{array}{l}87.1 \\
(12.4 \%)\end{array}$ & $\begin{array}{l}109.6 \\
(10.0 \%)\end{array}$ \\
\hline
\end{tabular}


En la figura 10 se muestran las relaciones entre el producto $V_{f} \times\left(l_{f} / d_{f}\right)$ y las principales propiedades mecánicas que definen el desempeño a flexión del CRFA. Tal como se esperaba, la resistencia al agrietamiento de tensión por flexión o módulo de ruptura $\left(f_{r}\right)$ y la deformación asociada $\left(\varepsilon_{r}\right)$ no varían significativamente al incrementar el contenido de fibras de acero (figuras 10a y 10b), pues a partir de este esfuerzo inicia la contribución de las fibras al concreto. Sin embargo, la resistencia máxima a tensión por flexión $\left(f_{\max }\right)$, la deformación asociada a $f_{\max }\left(\varepsilon_{\max }\right)$ y la tenacidad $(T)$ sí aumentan proporcionalmente con el contenido de fibras de acero (figuras 10c, 10d y 10f).

En las figuras $8 c, 9 b$ y $9 c$ se observa que algunos de los CRFA exhibieron endurecimiento por deformación, es decir, la resistencia máxima a tensión por flexión $\left(f_{\max }\right)$ fue mayor que la resistencia al agrietamiento de tensión por flexión $\left(f_{r}\right)$, debido a un mejor trabajo de la matriz cementante-fibra. Para analizar la tendencia de endurecimiento por deformación, en la figura 10e se muestran los datos del cociente $f_{\max } / f_{r^{\prime}}$ junto con una línea de tendencia bilineal. En la figura se observa que el endurecimiento por deformación se inicia para valor del producto $V_{f} \times\left(l_{f} / d_{f}\right)$ mayores que aproximadamente 50; para valores menores, la resistencia máxima está asociada a la resistencia de agrietamiento o módulo de ruptura.
En la figura 11 se muestran las relaciones entre el producto $V_{f} \times\left(l_{f} / d_{f}\right)$ y las resistencias residuales a flexión del CRFA. Tal como se esperaba, las resistencias residuales asociadas a deflexiones en el centro del claro equivalentes a $l_{f} / 24, l_{c} / 300 \mathrm{y} l_{c} / 150$, aumentan significativamente a medida que se incrementa la cantidad de fibras de acero (figuras 11a, 11b y 11c). En la figura 11 se observa que las curvas de tendencia lineal muestran una correlación adecuada con los datos medidos, tal como lo demuestran los valores cercanos a uno de los coeficientes de correlación.

\section{Ecuaciones propuestas}

A pesar que el ACI-318 (2011) permite el uso de fibras de acero en sustitución del refuerzo mínimo por cortante en vigas, el cual se dispone de forma convencional utilizando estribos de acero, dicho reglamento no especifica ecuaciones para estimar las propiedades mecánicas del CRFA. A partir de las tendencias de los resultados experimentales del estudio, se propone calcular las propiedades mecánicas promedio del CRFA utilizando las ecuaciones que se presentan en la tabla 6 . Las correlaciones se plantearon utilizando un formato que pueda ser fácilmente implantado en los reglamentos de diseño. Con base en las tendencias observadas, las propiedades mecánicas dependen del producto

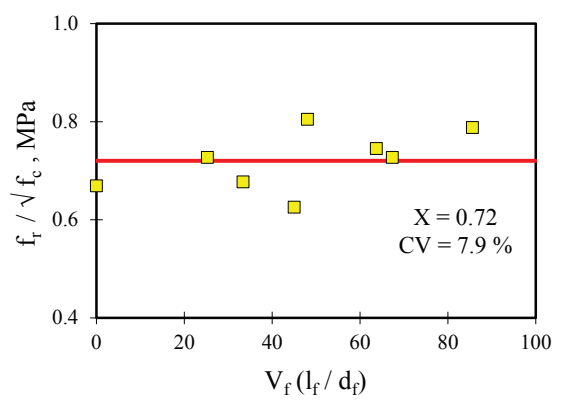

a) $\mathrm{fr}$

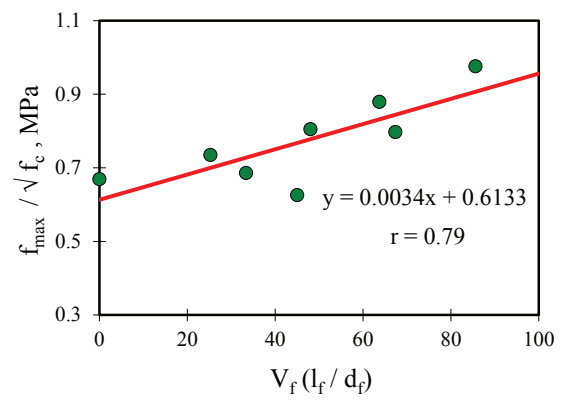

d) fmax

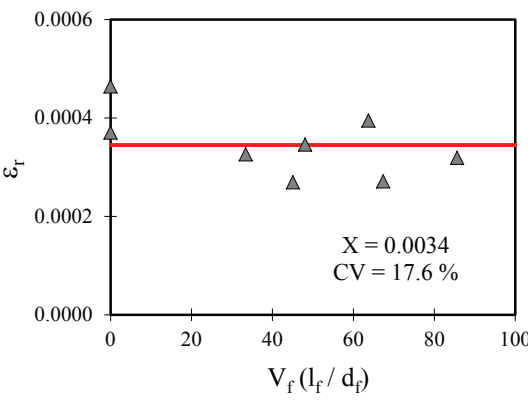

b) er

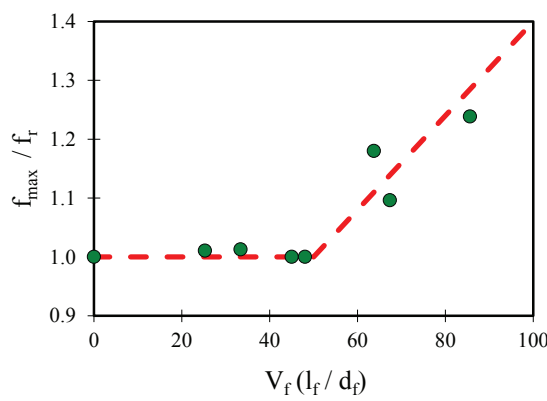

e) fmax

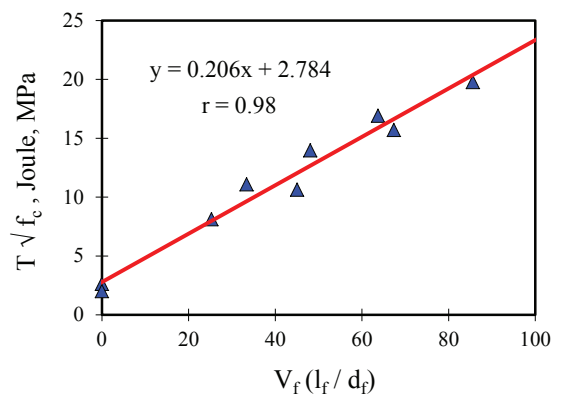

c) $\mathrm{T}$

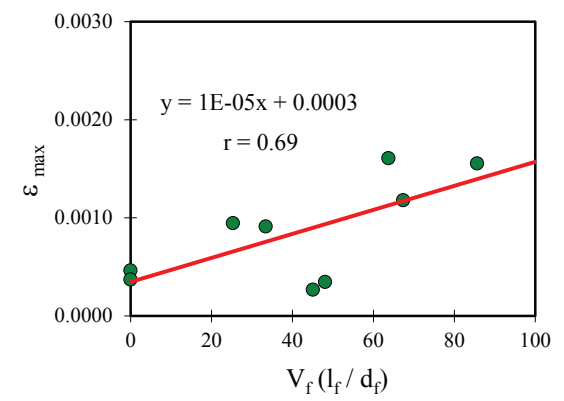

f) emax

Figura 10. Tendencias de las principales propiedades mecánicas a flexión del CRFA 


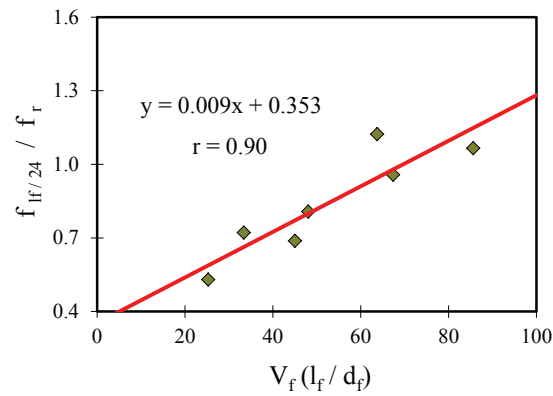

a) $\mathrm{flf} / 24 / \mathrm{fr}$

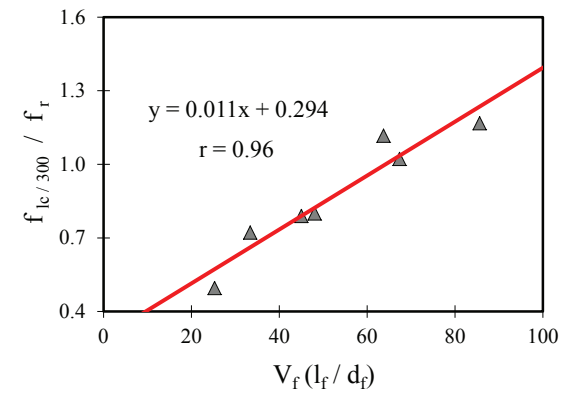

b) $\mathrm{flc} / 300 / \mathrm{fr}$

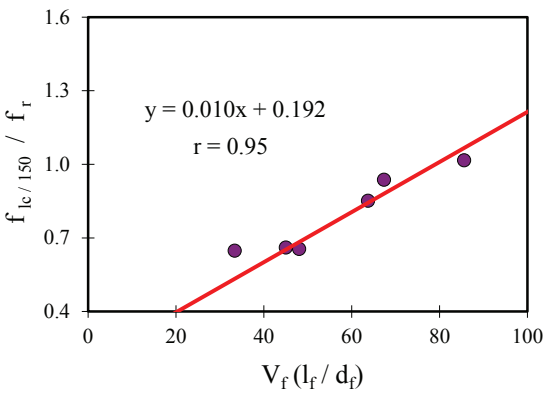

c) $\mathrm{flc} / 150 / \mathrm{fr}$

Figura 11. Tendencias de las propiedades mecánicas a flexión del CRFA

$V_{f} \times\left(l_{f} / d_{f}\right)$ (relación de volumen de fibras, $\%, \times$ relación de aspecto de la fibra) y/o la resistencia a compresión del concreto sin fibras $\left(f_{c 0}\right)$. En la tabla 6 se indica el valor del parámetro estadístico utilizado para la evaluación de la predicción. El valor definitivo de las constantes de las ecuaciones se debe establecer con base en los requisitos de probabilidad de excedencia que se especifican en cada reglamento.

\section{Limitaciones}

Las ecuaciones propuestas en el estudio pueden utilizarse para calcular las propiedades mecánicas promedio del concreto reforzado con fibras de acero (CRFA), que cumplan con las siguientes características:

Tipo de concreto:

Peso específico del concreto

(endurecido):

Resistencia a compresión del

concreto:

Resistencia a tensión de la fibra:

Tipo de anclaje:

Dosificación de fibras, $D_{f}$ :

peso normal

1800 a $2000 \mathrm{~kg} / \mathrm{m}^{3}$

$20 \mathrm{MPa}$ a $35 \mathrm{Mpa}$

$1100 \mathrm{MPa}$

con gancho

30 a $90 \mathrm{~kg} / \mathrm{m}^{3}$

Relación de aspecto de fibras, $l_{f} / d_{f}: 55$ a 80 .

\section{Conclusiones}

Para desarrollar ayudas de diseño que promuevan el uso del concreto reforzado con fibras de acero (CRFA) en muros de concreto de vivienda de interés social (VIS), en el artículo se han presentado los resultados de un extenso programa experimental que incluyó el ensayo de 88 especímenes en forma de cilindros y 40 especímenes en forma de vigas. A partir de las tendencias de los resultados experimentales, se propusieron correlaciones numéricas para estimar las propiedades mecánicas básicas del CRFA (resistencia máxima y deformación en compresión, módulo de elasticidad, relación de Poisson y resistencia a tensión indirecta), y las propiedades que caracterizan el desempeño a flexión (resistencia de agrietamiento, capacidad de deformación y capacidad de disipación de energía o tenacidad). Las principales conclusiones de la investigación se presentan a continuación:

\section{Características básicas}

La manejabilidad del concreto disminuyó significativamente a medida que incrementó la cantidad de fibras en el concreto, especialmente cuando se utilizan fibras tipo con longitud igual a $60 \mathrm{~mm}$.

El peso específico en estado endurecido no varía significativamente al aumentar el contenido de fibras, pues la disminución de agregado grueso se equilibra con la adición de fibras de acero.

\section{Comportamiento en compresión y en tensión}

A medida que se incrementa el contenido de fibras de acero, la resistencia a compresión del concreto disminuye a un valor casi constante $\left(f_{c} / f_{c o}=0.87\right)$ y su capacidad de deformación en compresión aumenta.

El módulo de elasticidad disminuye a medida que se incrementa el contenido de fibras de acero. Esta tendencia se genera por la disminución de agregado grueso (sustitución de agregado grueso por fibra de acero), y por el aumento del contenido de aire en la mezcla, el cual crea menor adherencia entre la matriz cementante y la fibra. El aumento de aire se origina porque éste queda atrapado por la disminución de revenimiento del concreto o porque se introduce en el momento del mezclado de las fibras.

En general, la fibra de acero le proporciona un efecto de confinamiento al concreto en compresión y, por tanto, a medida que incrementa el contenido de fibras 


\begin{tabular}{lcc}
\hline Ecuación propuesta & Unidad & $\begin{array}{c}\text { Parámetro } \\
\text { estadístico }\end{array}$ \\
\hline$\varepsilon_{0}=0.0037+0.00002\left(V_{f} \times\left(l_{f} / d_{f}\right)\right)$ & --- & $r=0.66$ \\
$E_{c}=\left[2509-11.4\left(V_{f} \times\left(l_{f} / d_{f}\right)\right)\right] \sqrt{f_{c}}$ & $\mathrm{MPa}$ & $r=0.88$ \\
$f_{t}=\left[0.34+0.0052\left(V_{f} \times\left(l_{f} / d_{f}\right)\right)\right] \sqrt{f_{c}}$ & $\mathrm{MPa}$ & $r=0.91$ \\
$f_{r}=0.72 \sqrt{f_{c}}$ & $\mathrm{MPa}$ & $\mathrm{CV}=7.9 \%$ \\
$f_{\max }=\left[0.61+0.0034\left(V_{f} \times\left(l_{f} / d_{f}\right)\right)\right] \sqrt{f_{c}}$ & $\mathrm{MPa}$ & $r=0.79$ \\
$T=\left[2.78+0.21\left(V_{f} \times\left(l_{f} / d_{f}\right)\right)\right] \sqrt{f_{c}}$ & $\mathrm{Joule}$ & $r=0.98$ \\
$f_{t / f / 24}=\left[0.35+0.01\left(V_{f} \times\left(l_{f} / d_{f}\right)\right)\right] \sqrt{f_{c}}$ & $\mathrm{MPa}$ & $r=0.90$ \\
$f_{l / l 300}=\left[0.29+0.01\left(V_{f} \times\left(l_{f} / d_{f}\right)\right)\right] \sqrt{f_{c}}$ & $\mathrm{MPa}$ & $r=0.96$ \\
$f_{l c / 150}=\left[0.19+0.01\left(V_{f} \times\left(l_{f} / d_{f}\right)\right)\right] \sqrt{f_{c}}$ & $\mathrm{MPa}$ & $r=0.95$ \\
\hline
\end{tabular}

Tabla 6. Ecuaciones propuestas para calcular las propiedades mecánicas del CRFA de acero, la relación de Poisson disminuye a un valor casi constante $(v=0.15)$.

En términos generales, la fibra de acero genera que el concreto se vuelva ligeramente menos resistente, pero más dúctil cuando éste se somete a fuerzas axiales de compresión.

En cuanto al ensayo de compresión diametral, tal como se esperaba, la resistencia a tensión indirecta del concreto aumenta proporcionalmente con la cantidad de fibras de acero.

\section{Desempeño en flexión}

La prueba de flexión es considerada como una de las más representativas para el CRFA, pues a partir de ella se puede estimar la capacidad de deformación que la fibra le proporciona al concreto después de alcanzar el agrietamiento inicial por flexión. Adicionalmente, a partir de los resultados medidos durante esta prueba, se determina la tenacidad del CRFA; es decir, la capacidad de absorción de energía del material.

Tal como se esperaba, la resistencia al agrietamiento de tensión por flexión o módulo de ruptura y la deformación unitaria asociada, no varían significativamente al incrementar el contenido de fibras de acero, pues a partir de este esfuerzo inicia la contribución de las fibras al concreto. Sin embargo, la resistencia máxima a tensión por flexión y su deformación unitaria asociada, así como la tenacidad, sí aumentan proporcionalmente con el contenido de fibras de acero.

De forma similar, se observó que las resistencias residuales aumentaron significativamente a medida que se incrementó la cantidad de fibras de acero.
El endurecimiento por deformación se observó en CRFA con valores del producto $V_{f} \times\left(l_{f} / d_{f}\right)$ mayores que aproximadamente 50. Este comportamiento demuestra un mejor trabajo de la matriz cementante-fibra, el cual está asociado principalmente a una mejor adherencia entre ambos componentes.

\section{Ecuaciones propuestas}

Las correlaciones numéricas se plantearon utilizando un formato que pueda ser fácilmente implantado en los reglamentos de diseño. Por ejemplo, las propiedades mecánicas dependen del producto $V_{f} \times\left(l_{f} / d_{f}\right)$ (relación de volumen de fibras, $\%, \times$ relación de aspecto de la fibra) y/o la resistencia a compresión del concreto sin fibras $\left(f_{c 0}\right)$. El valor definitivo de las constantes de las ecuaciones se debe establecer con base en los requisitos de probabilidad de excedencia que se especifican en cada reglamento.

Las recomendaciones del reglamento ACI-318 (2008) para refuerzo mínimo a cortante de vigas sólo dependen del valor de la dosificación de fibras $\left(D_{f}\right.$ o su equivalente $V_{f}$ ). Tomando en cuenta las tendencias de las propiedades medidas de CRFA, las ecuaciones propuestas en este estudio dependen tanto de la dosificación $\left(V_{f}\right)$ como de la relación de aspecto de la fibra $\left(l_{f} / d_{f}\right)$.

Considerando las características particulares de muros de concreto para VIS, las demandas sísmicas en este tipo de estructuras y las propiedades mecánicas del CRFA, los muros de concreto con fibras de acero tienen gran potencial para su utilización en la construcción de VIS. 


\section{Referencias}

Adebar P., Mindess S., Pierr D., Olund B. Shear Tests of Fiber Concrete Beams Without Stirrups. ACI Structural Journal, volumen 94 (número 1), 1997: 68-76.

ACI-Comité 318. Building Code Requirements for Structural Concrete and Commentary (ACI 318-08), American Concrete Institute, Farmington Hills, MI, EUA, 2011.

ACI-Comite 544. Report on the Physical Properties and Durability of Fiber-Reinforced Concrete (ACI 544.5R-10), American Concrete Institute, Farmington Hills, MI, EUA, 2010.

ACI-Comité 544. State-of-the-Art Report on Fiber Reinforced Concrete (ACI 544.1R-96), American Concrete Institute, Farmington Hills, MI, EUA, 1996.

ASTM-C-39. Standard Test Method for Compressive Strength of Cylindrical Concrete Specimens, Libro anual, EUA, 1999, p. 5.

ASTM-C-469. Standard Test Method for Static Modulus of Elasticity and Poisson's Ratio of Concrete in Compression, Libro anual, EUA, 1994, p. 4.

ASTM-C-496. Standard Test Method for Splitting Tensile Strength of Cylindrical Concrete Specimens, Libro anual, EUA, 1996, p. 4.

ASTM-C-1609. Standard Test Method for Flexural Performance of Fiber-Reinforced Concrete (Using Beam with Third-Point Loading), Libro anual, EUA, 2010, p. 9.

Ávila O., Carrillo J., Alcocer S. Rehabilitación de muros de concreto usando CRFA: Ensayos en mesa vibradora. Concreto y Cemento: Investigación y Desarrollo, volumen 2 (número 2), 2011: 2-17.

Batson G., Terry T., Change M. Fiber Reinforced Concrete Beams Subjected to Combined Bending and Torsion, en: International Symposium of Fiber Reinforced Concrete, Publicación SP81, American Concrete Institute, Detroit, EUA, 1984, pp. 51-68.

Carrillo J. y Alcocer S. Backbone Model for Performance Based Seismic Design of RC Walls for Low-Rise Housing. Earthquake Spectra, volumen 28 (número 3), 2012: 943-964.

Craig R. Flexural Behavior and Design of Reinforced Fiber Concrete Members, Fiber Reinforced Concrete Properties and Applications, Publicación SP-105, American Concrete Institute, Detroit, EUA, 1987, pp. 517-563.

Dinh H., Parra-Montesinos G. y Wight J. Shear Behavior of Steel Fiber-Reinforced Concrete Beams Without Stirrup Reinforcement. ACI Structural Journal, volumen 107 (número 5), 2010: 597-606.
Jindal R. Shear Moment Capacities of Steel Fiber Reinforced Concrete Beams, en: International Symposium of Fiber Reinforced Concrete, Publicación SP-81, American Concrete Institute, Detroit, EUA, 1984, pp. 1-16.

Kalman D. Use of Steel Fiber Reinforced Concrete for Blast Resistant Design, tesis (maestría), Manhattan, Kansas, EUA, Kansas State University, 2010.

Kwak Y., Eberhard M., Kim W., Kim J. Shear Strength of Steel Fiber-Reinforced Concrete Beams Without Stirrups. ACI Structural Journal, volumen 99 (número 4), 2002: 530-538.

Miao B., Chern J., Yang Ch. Influences of Fiber Content on Properties of Self-Compacting Steel Fiber Reinforced Concrete. Journal of the Chinese Institute of Engineers, volumen 26 (número 4), 2003: 523-530.

Narayana R. y Darwish I. Use of Steel Fibers as Shear Reinforcement. ACI Structural Journal, volumen 84 (número 3), 1987: 216-227.

Parra-Montesinos G. Shear Strength of Beams with Deformed Steel Fibers. Journal of Concrete International, volumen 28 (número 11), 2006: 57-66.

Parra-Montesinos G. High-Performance Fiber-Reinforced Cement Composites: an Alternative for Seismic Design of Structures. ACI Structural Journal, volumen 102 (número 5), 2005: 668-675.

Turmo J., Banthia N., Gettu R., Barragán B. Estudio del comportamiento de vigas de hormigón reforzado con fibras. Revista Materiales de Construcción, volumen 58 (número 292), 2008: 5-13.

\section{Este artículo se cita:}

Citación estilo Chicago

Carrillo, Julián, William Aperador, Giovanni González. Correlaciones entre las propiedades mecánicas del concreto reforzado con fibras de acero. Ingeniería Investigación y Tecnología, XIV, 03 (2013): 437-452.

\section{Citación estilo ISO 690}

Carrillo J., Aperador W., González G. Correlaciones entre las propiedades mecánicas del concreto reforzado con fibras de acero. Ingeniería Investigación y Tecnología, volumen XIV (número 3), julio-septiembre 2013: 437-452. 


\section{Semblanza de autores}

Julián Carrillo. Obtuvo los títulos de ingeniero civil en 2002 por la Universidad Militar Nueva Granada, UMNG, Colombia, el de maestro en ingeniería civil (área de estructuras y sísmica) en 2004 por la Universidad de los Andes, Colombia, y el de doctor en ingeniería (área de estructuras) en 2010 por la Universidad Nacional Autónoma de México, UNAM. Actualmente es profesor y director del Grupo de Investigación de Estructuras y Sísmica de la UMNG. Es miembro de tres comités: del American Concrete Institute, ACI: 314, Diseño simplificado de edificios; 369, Reparación y rehabilitación sísmica; y 374, Diseño sísmico basado en desempeño de edificios de concreto.

William Aperador. Obtuvo los títulos de físico en 2003 y el de maestro en metalurgia y ciencia de los materiales en 2006 por la Universidad Pedagógica y Tecnológica de Colombia y el de doctor en ingeniería de los materiales en 2009 por la Universidad del Valle, Colombia. Realizó una pasantía internacional en el centro nacional de investigaciones metalúrgicas (CENIM), en Madrid, España, en el Laboratorio de Ingeniería de Materiales y Durabilidad. Actualmente es profesor e investigador del Grupo de Investigación GMCDP-Volta. Ha sido autor y coautor de más de 80 artículos y ha presentado 40 ponencias internacionales y 38 comunicaciones nacionales.

Giovanni González. Obtuvo el título de ingeniero civil en 1987 por la Universidad de la Salle, Colombia. Actualmente realiza estudios de especialización y maestría en la Escuela Colombiana de Ingeniería Julio Garavito. Actualmente es profesor, investigador y jefe del Área de Estructuras del Departamento de Ingeniería Civil de la Universidad Militar Nueva Granada, UMNG, Colombia. Es asesor estructural de varias empresas constructoras en Colombia y es miembro de la Asociación Colombiana de Ingenieros Estructurales, ACIES. 